\title{
Зондирование космической плазмы радиоимпульсами пульсаров в проекте "Радиоастрон"
}

\author{
Е.Н. Фадеев ${ }^{1}$, А.С. Андрианов ${ }^{1}$, В.А. Зуга ${ }^{1}$, М.В. Попов ${ }^{1}$, А.Г. Рудницкий두 Т.В. Смирнова ${ }^{2}$, \\ B.A. Согласнов ${ }^{1}$, В.И. Шишов ${ }^{2}$ \\ 1 Астрокосмический центр Физического института им. П.Н. Лебедева Российской академии наук, \\ Москва, Россия \\ fadeev@asc.rssi.ru \\ 2 Пущинская радиоастрономическая обсерватория АКЦ ФИАН, Пущино, Московская область, Россия \\ tania@prao.ru
}

Поступила в редакцию 15 ноября 2017 г.

Аннотация. Наземно-космический интерферометр "Радиоастрон” обладает непревзойденной разрешающей способностью. С его помощью были определены размеры кружков рассеяния в направлении 6 пульсаров: В0329+54, В0525+21, В1641-45, В1749-28, В1919+21, В1933+16. Наблюдения проводились на частотах 324 и 1668 МГц. Нами были определены также характерные масштабы мерцаний по времени и по частоте. Было показано, что в направлении всех пульсаров межзвездная среда распределена неоднородно, и были определены расстояния до эффективных рассеивающих экранов.

В результате анализа вторичного спектра пульсара В1933+16 на частоте 1668 МГц были выявлены две параболические дуги, которые соответствуют двум рассеивающим экранам.

PROBING OF COSMIC PLASMA USING RADIOASTRON VLBI OBSERVATIONS OF RADIO PULSARS, by E.N. Fadeev, A.S. Andrianov, V.A. Zuga, M.V. Popov, A.G. Rudnitskiy, T.V. Smirnova, V.A. Soglasnov, V.I. Shishov. The RadioAstron ground-space interferometer has an unsurpassed angular resolution. By means of RadioAstron we determined the angular size of the scatter-broadened image of six radio pulsars: $\mathrm{B} 0329+54$, B0525+21, B1641-45, B1749-28, B1919+21, B1933+16. We made observations at 324 and $1668 \mathrm{MHz}$. At the same time, we measured other parameters of pulsars scintillation, such as scintillation time and temporal broadening of pulse. We showed that the interstellar medium in the direction of all pulsars is nonuniform, and we obtained distances to scattering screens.

In the secondary spectrum of $\mathrm{B} 1933+16$ at $1668 \mathrm{MHz}$ we revealed 2 parabolic arcs that correspond to 2 scattering screens.

Ключевые слова: пульсары - межзвездная среда

\section{1 Введение}

Пульсары, в силу своей природы, являются идеальными объектами для исследования ионизированной межзвездной плазмы. Благодаря импульсному характеру излучения и малым угловым размерам пульсаров мы можем исследовать различные эффекты влияния межзвездной плазмы на принимаемое излучение. Основным эффектом является рассеяние. При этом максимум излучения пульсаров приходится на метровый диапазон электромагнитных волн, где рассеяние достаточно заметно. 
K наблюдаемым эффектам рассеяния можно отнести следующие: увеличение углового размера пульсаров; узкополосные изменения интенсивности излучения по частоте и времени - мерцания. Размер диска рассеяния обычно не удается измерить с помощью наземных интерферометров, поскольку их диаграмма направленности существенно больше него. С помощью наземно-космического интерферометра "Радиоастрон” мы смогли измерить диаметры дисков рассеяния ряда пульсаров (Попов и др., 2016; Андрианов и др., 2017; Попов и др., 2017; Шишов и др., 2017).

Сочетая анализ временных и частотных параметров мерцаний с измерением дисков рассеяния, мы смогли определить распределение межзвездной среды в направлении исследуемых пульсаров. Дополнительную информацию о распределении межзвездной среды дает анализ вторичных спектров пульсаров.

\section{2 Наблюдения и первичная обработка данных}

Все результаты были получены с помощью наземно-космического радиоинтерферометра "Радиоастрон". Космический аппарат "Спектр-Р" с 10-метровым радиотелескопом на борту был запущен 18 июля 2011 года на высокоапогейную орбиту. Благодаря тому, что в апогее космический аппарат удаляется более чем на 300000 км, возможны наблюдения с экстремально высоким разрешением вплоть до долей миллисекунды дуги. В нашей работе мы использовали два из четырех возможных на космическом радиотелескопе (КРТ) диапазона наблюдений: 1.668 ГГц и 324 МГц. Ширина полосы приемника на 324 МГц составляет 16 МГц и 32 МГц - на 1.668 ГГц (Кардашев и др., 2013).

КРТ обладает рядом особенностей, которые требуется принимать во внимание при работе. В силу ограничений теплового режима на борту непрерывная работа КРТ ограничена 1-2 часами. КРТ может одновременно проводить наблюдения в двух частотных диапазонах. Такие сеансы наблюдений проводились, например, при исследовании пульсара В1933+16. При этом для наземной поддержки требуется отдельный набор телескопов для каждого спектрального диапазона.

Данные КРТ и наземных телескопов собираются вместе и обрабатываются с помощью коррелятора АКЦ ФИАН. Для компенсации размытия сигнала, вызванного дисперсией в межзвездной среде, используется алгоритм некогерентной дедисперсии. Для повышения отношения сигнал-шум для корреляции используется только сигнал в определенном окне, выделяющем импульс пульсара (Лихачев и др., 2017). Отдельно производится обработка сигнала вне импульса с целью калибровки полосы пропускания и устранения помех.

\section{3 Теоретические соотношения и результаты}

\section{1 Кросс-спектр электрического поля}

На выходе коррелятор выдает произведение электрических полей на двух антеннах А и В, которое обычно называют кросс-спектром, и автоспектры для каждой из антенн:

$$
I(\boldsymbol{\rho}, \boldsymbol{\rho}+b, \nu, t)=E_{A}(\boldsymbol{\rho}, \nu, t) E_{B}^{*}(\boldsymbol{\rho}+\mathbf{b}, \nu, t) .
$$

Здесь $\boldsymbol{\rho}$ - пространственная координата в uv-плоскости, $\mathbf{b}$ - проекция базы на uv-полоскость, $\nu$ - частота, $t$ - время. Авто(кросс)-спектры для каждой пары антенн являются функцией не только частоты, но и времени. Такой меняющийся во времени спектр обычно называют динамическим спектром (рис. 1). В результате мерцаний энергия, переносимая излучением, усиливается и ослабляется в ограниченных участках по частоте и по времени. Характерный масштаб этих областей по частоте называют полосой декорреляции $\Delta \nu_{d}$, а по оси времени - временем мерцаний $\Delta t_{s c}$.

Для определения значений $\Delta \nu_{d}$ и $\Delta t_{s c}$ мы вычисляли двумерную автокорреляционную функцию динамического спектра и рассматривали сечения этой функции вдоль осей задержки по частоте и по времени соответственно. Обычно сечение в своей средней части хорошо описывается гауссианой или экспоненциальной функцией, и в качестве искомых характеристик принимается полуширина 


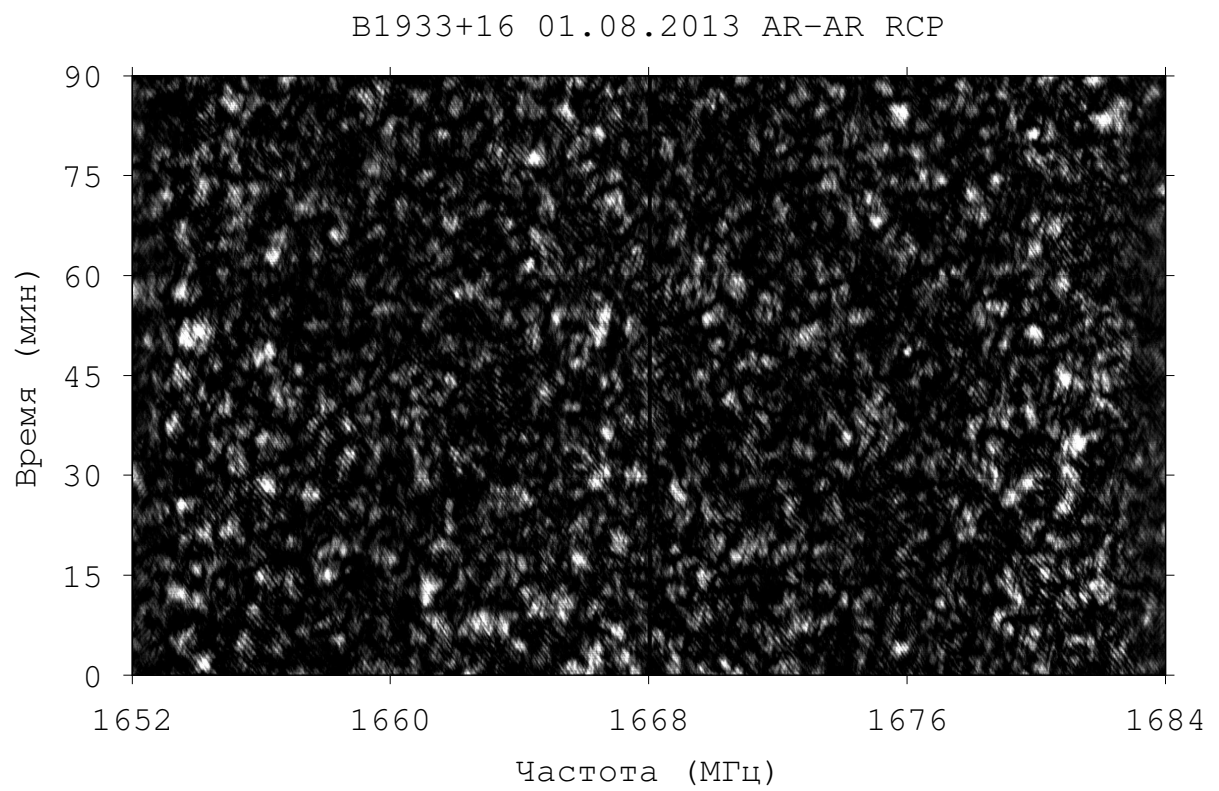

Рис. 1. Динамический автоспектр пульсара В1933+16, полученный на телескопе обсерватории Аресибо 01.08.2013 на частоте 1.667 ГГц $(18 \mathrm{~cm})$. Полоса приемника состоит из двух субполос по 16 МГц каждая. Продолжительность наблюдательного сеанса 90 минут

Таблица 1. Измеренные характеристики мерцаний пульсаров

\begin{tabular}{|c|c|c|c|c|c|c|c|}
\hline Пульсар & Частота $($ МГц $)$ & $\Delta t_{s c}$ (c) & $\Delta \nu_{d}(\mathrm{\kappa} Г ц)$ & $\tau_{s c}(\mathrm{MKC})$ & $\theta_{H}, 10^{-3 \prime \prime}$ & $D$ (кпк) & $d(\mathrm{K \Pi K)}$ \\
\hline B0329+54 & 324 & 114 & 15 & 12.1 & 5.0 & 1.03 & 0.6 \\
\hline B $0525+21$ & 1668 & 160 & 3900 & 0.041 & 0.066 & 1.6 & 1.4 \\
\hline B1641-45 & 1668 & 0.20 & 0.062 & 2600 & 27 & 4.9 & 3.0 \\
\hline B1749-28 & 1668 & 220 & 410 & 0.31 & 0.5 & 1.3 & 0.95 \\
\hline B $1919+21$ & 324 & 290 & 330 & 0.48 & 1.7 & 1 & $1.4 \times 10^{-4} ; 0.44$ \\
\hline B1933+16 & 1668 & 41.6 & 50.4 & 3.2 & 0.84 & 3.7 & $2.7[1 ; 2.9]$ \\
\hline B1933+16 & 324 & - & 0.25 & 600 & 12.3 & 3.7 & 2.6 \\
\hline
\end{tabular}

соответствующей функции на половине максимума корреляционной функции. Полученные величины приведены в таблице 1 .

Принимая во внимание известное соотношение $2 \pi \Delta \nu_{d} \tau_{s c}=1$ (Саттон, 1971), мы получаем величину времени рассеяния $\tau_{s c}$ - характерного времени, на которое растягивается изначально короткий импульс пульсара вследствие рассеяния.

\section{2 Интерферометрическая видность}

Под интерферометрической видностью понимают результат двумерного преобразования Фурье, переводящий динамический спектр из координат частота-время в сопряженные с ними координаты задержка-частота интерференции:

$$
V(\boldsymbol{\rho}, \boldsymbol{\rho}+b, \tau, f)=\mathfrak{F}_{\nu \rightarrow \tau}^{-1}\left[\mathfrak{F}_{\nu \rightarrow \tau}[I(\boldsymbol{\rho}, \boldsymbol{\rho}+b, \nu, t)]\right] .
$$

Функция видности на малых проекциях базы, при которых еще не разрешается кружок рассеяния, имеет пик в начале координат и протяженную часть. По мере увеличения проекции базы амплитуда 
центрального пика уменьшается до тех пор, пока не перестает выделяться среди множества подобных маленьких пиков, расположенных в пределах протяженной части (рис. 2). Это можно трактовать сле-

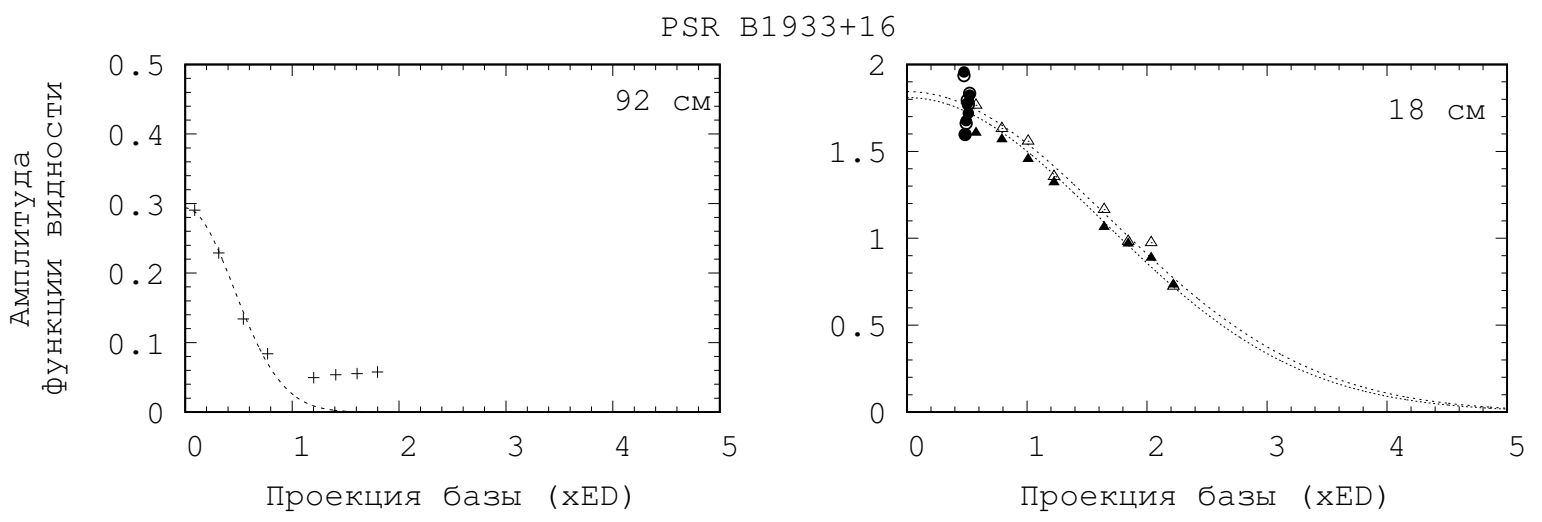

Рис. 2. Зависимость амплитуды функции видности от величины проекции базы в двух диапазонах для пульсара В1933+16. Наблюдения на 92 см проводились на базе "Радиоастрон” - Вестерборк. Примерно на середине наблюдательного сеанса диск рассеяния оказался полностью разрешенным. В диапазоне 18 см указаны результаты наблюдений на базах "Радиоастрон" - Аресибо (треугольники) и Аресибо - Светлое (кружки). Закрашенные фигуры соответствуют верхней субполосе, незакрашенные - нижней. В этом диапазоне кружок рассеяния до конца не был разрешен даже на самых больших базах

дующим образом. На малых проекциях базы кружок рассеяния остается неразрешенным источником. По мере увеличения проекции базы кружок начинает разрешаться; диаграмма направленности интерферометра становится меньше кружка рассеяния, вследствие чего интерференционный отклик ослабевает. При дальнейшем увеличении проекции базы основной вклад в интерференционный отклик дает субструктура - отдельные увеличения яркости внутри кружка рассеяния (Гвинн и др., 2016).

В работе (Гвинн и др., 1988) показано, что зависимость амплитуды функции видности от проекции базы имеет вид

$$
V(b)=V_{0} \exp \left\{-\frac{1}{2}\left[\frac{\pi}{\sqrt{2 \ln 2}} \frac{b \theta_{H}}{\lambda}\right]^{\alpha-2}\right\},
$$

где $\theta_{H}$ - угловой размер кружка рассеяния, $\lambda$ - длина волны наблюдения, $\alpha$ - показатель степени спектра неоднородности плазмы, который обычно близок к значению колмогоровского спектра $11 / 3$. Для построения такой зависимости требуется калибровка значений амплитуды видности для всех пар антенн на всех базах. Эта задача может оказаться достаточно сложной. В работе (Шишов и др., 2017) был предложен другой способ оценки амплитуды функции видности, который мы также использовали.

С помощью изложенного метода мы измерили размеры кружков рассеяния для 6 пульсаров. Результаты приведены в таблице 1.

\section{3 Структура межзвездной среды}

Угловой размер кружка рассеяния и время рассеяния зависят от распределения межзвездной среды на луче зрения. В работе (Бриттон и др., 1998) приведены простейшие частные случаи. Для среды, в которой рассеивающая материя распределена равномерно, справедливо соотношение

$$
\theta_{H, u}^{2}=16 \ln 2 c \tau_{s c} / D,
$$


где $D$ - расстояние до пульсара, а $c$ - скорость света. Во всех наших экспериментах это соотношение не выполнялось, что говорит о том, что межзвездная среда распределена неравномерно. Другим важным частным случаем является такой, в котором вся рассеивающая среда расположена в области, толщина которой много меньше расстояния до пульсара (рассеивающий экран). Тогда

$$
\theta_{H, s}^{2}=\frac{8 \ln 2 c \tau_{s c}}{D} \frac{s}{1-s}=\frac{\theta_{H, u}^{2}}{2} \frac{s}{1-s},
$$

где $s=(D-d) / D$ - расстояние от пульсара до экрана в долях $D$. Измеренные таким образом расстояния от наблюдателя до экрана $d$ приведены в таблице 1 .

\section{4 Вторичные спектры}

Особый интерес имеет анализ вторичных спектров, которые являются двумерным преобразованием Фурье от динамических спектров (рис. 3). Во вторичных спектрах некоторых пульсаров мы можем

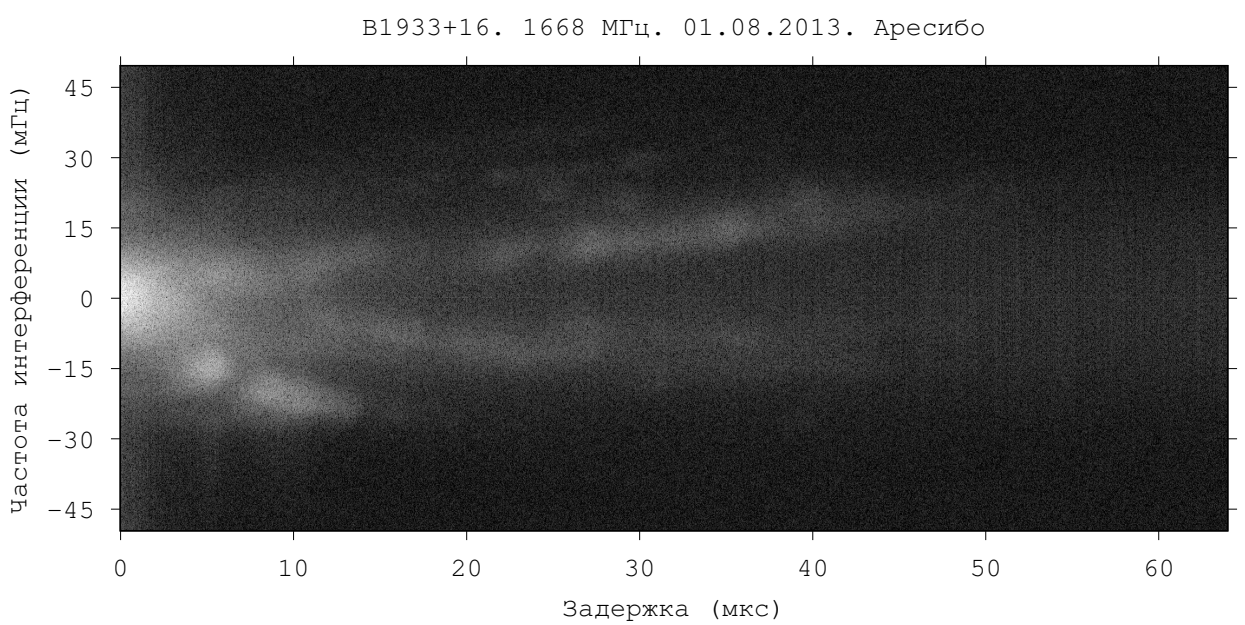

Рис. 3. Вторичный спектр пульсара В1933+16, полученный на телескопе обсерватории Аресибо 01.08.2013 на частоте 1.668 ГГц (18 см). Две ярко выраженные параболические дуги соответствуют двум экранам. Принимая расстояние до пульсара равным 3.7 кпк, получаем для внутренней дуги $s=0.71$, а для внешней $s=0.22$, что соответствует положению экранов на расстоянии 1 и 2.9 кпк от Солнца

видеть избыток мощности вдоль параболических дуг с вершинами вблизи начала координат, иногда вложенных одна в другую, или даже в виде набора обратных арок, вершины которых лежат вдоль главной параболы (Кордс и др., 2006). Каждая из этих параболических структур возникает при рассеянии излучения на тонком экране, а ее кривизна $а$ связана с расстоянием до экрана следующим образом:

$$
a=\frac{D \lambda^{2}}{2 c\left|\mathbf{v}_{p \perp}\right|^{2}} \frac{s}{1-s} .
$$

Здесь $\mathbf{v}_{p \perp}$ имеет смысл скорости, с которой движется луч зрения относительно некоторой точки среды в плоскости, перпендикулярной лучу зрения. Эту величину можно выразить через тангенциальную скорость движения пульсара $\mathbf{v}_{p \perp}$, наблюдателя $\mathbf{v}_{o \perp}$ и экрана $\mathbf{v}_{s \perp}$, как

$$
\mathbf{v}_{p \perp}=(1-s) \mathbf{v}_{p \perp}+s \mathbf{v}_{o \perp}+\mathbf{v}_{s \perp}
$$


В нашем исследовании параболические дуги были обнаружены только у пульсара B1933+16. Принимая во внимание высокую пространственную скорость пульсара (220 км/с), мы пренебрегли скоростью движения среды и наблюдателя. Полученные расстояния до рассеивающих экранов составили 1 и 2.9 кпк. Положение второго экрана хорошо согласуется со значением, полученным при анализе кружка рассеяния.

\section{4 Заключение}

В результате экспериментов, проведенных с помощью наземно-космического интерферометра "Радиоастрон”, нами были определены размеры кружков рассеяния 6 пульсаров. Было показано, что рассеяние происходит главным образом в узких слоях межзвездной плазмы - рассеивающих экранах, расстояние до которых было измерено.

Проект "Радиоастрон" осуществляется Астрокосмическим центром Физического института им. П.Н. Лебедева Российской академии наук и Научно-производственным объединением им. С.А. Лавочкина по контракту с Российским космическим агентством совместно с многими научно-техническими организациями в России и других странах.

Представленные результаты частично основаны на наблюдениях, выполненных на радиотелескопах ИПА РАН (Федеральное государственное бюджетное учреждение науки Институт прикладной астрономии Российской академии наук).

Представленные исследования частично основаны на результатах наблюдений Европейской РСДБ-сети (European VLBI Network), а также на результатах наблюдений: радиотелескопа обсерватории Аресибо, телескопов Национальной радиоастрономической обсерватории (NRAO, CША) и телескопов Национального австралийского комплекса (ATNF, Австралия и Университет Тасмании).

Данная работа поддержана грантом РФФИ 1602-00954.

\section{Литература}

Андрианов А.С., Смирнова Т.В., Шишов В.И., Гвин К., Попов М.В. // Астрон. журн. 2017. Т. 94. C. 516.

Бриттон и др. (Britton M.C., Gwinn C.R., Ojeda M.J.) // Astrophys. J. 1998. V. 501. Issue 1. P. L101. Гвинн и др. (Gwinn C.R., Bartel N., Cordes J.M., Wolszczan A., Mutel R.L.) // Astrophys. J. 1988. V. 334. P. L13.

Гвинн и др. (Gwinn C.R., Popov M.V., Bartel N., et al.) // Astrophys. J. 2016. V. 822. Issue 2. P. 96. Кардашев Н.С., Хартов В.В., Абрамов В.В. и др. // Астрон. журн. 2013. Т. 90. № 3. С. 179.

Кордс и др. (Cordes J.M., Rickett B.J., Stinebring D.R., Coles W.A.) // Astrophys. J. 2006. V. 637. Issue 1. P. 346.

Лихачев и др. (Likhachev S.F., Kostenko V.I., Girin I.A., Andrianov A.S., Zharov V.E., Rudnitskiy A.G.) // J. Astron. Instrum. 2017. V. 6. Issue 3. id. 1750004-131.

Попов М.В., Андрианов А.С., Бартель Н., Гвинн К., Джоши Б.Ч. и др. // Астрон. журн. 2016. Т. 93. C. 778 .

Попов и др. (Popov M.V., Bartel N., Gwinn C.R., Johnson M.D., et al.) // Mon. Not. Roy. Astron. Soc. 2017. V. 465. P. 978.

Саттон (Sutton J.M.) // Mon. Not. Roy. Astron. Soc. 1971. V. 155. P. 51.

Шишов и др. (Shishov V.I., Smirnova T.V., Gwinn C.R., Andrianov A.S., Popov M.V., et al.) // Mon. Not. Roy. Astron. Soc. 2017. V. 468. Issue 3. P. 3709. 